\title{
Characterisation of children hospitalised with pneumonia in central Vietnam: a prospective study
}

\author{
Phuong T.K. Nguyen (1) ${ }^{1,2}$, Hoang T. Tran², Dominic A. Fitzgerald ${ }^{1,3}$, \\ Thach S. Tran ${ }^{4}$, Stephen M. Graham $\mathbb{1}^{5}$ and Ben J. Marais ${ }^{1}$
}

Affiliations: ${ }^{1}$ Discipline of Child and Adolescent Health, Sydney Medical School, The Children's Hospital at Westmead, The University of Sydney, Sydney, Australia. ${ }^{2}$ Da Nang Hospital for Women and Children, Da Nang, Vietnam. ${ }^{3}$ Dept of Respiratory Medicine, The Children's Hospital at Westmead, Sydney, Australia. ${ }^{4}$ Clinical Studies and Epidemiology, Bone Biology Division, Garvan Institute of Medical Research, Darlinghurst, Australia. ${ }^{5}$ Centre for International Child Health, University of Melbourne and Murdoch Children's Research Institute, Royal Children's Hospital, Melbourne, Australia.

Correspondence: Phuong T.K. Nguyen, Respiratory Dept, Da Nang Hospital for Women and Children, 402 Le Van Hien Street, Ngu Hanh Son District, Da Nang, Vietnam. E-mail: thng5150वuni.sydney.edu.au

@ERSpublications

Many child "pneumonia" admissions in central Vietnam do not meet WHO case management criteria for hospitalisation or intravenous antibiotics. Accurate aetiological diagnosis is challenging and astute clinical judgement should guide optimal management. http://ow.ly/vkHV30ojiOY

Cite this article as: Nguyen PTK, Tran HT, Fitzgerald DA, et al. Characterisation of children hospitalised with pneumonia in central Vietnam: a prospective study. Eur Respir J 2019; 54: 1802256 [https://doi.org/ 10.1183/13993003.02256-2018].

ABSTRACT Pneumonia is the most common reason for paediatric hospital admission in Vietnam. The potential value of using the World Health Organization (WHO) case management approach in Vietnam has not been documented.

We performed a prospective descriptive study of all children (2-59 months) admitted with "pneumonia" (as assessed by the admitting clinician) to the Da Nang Hospital for Women and Children to characterise their disease profiles and assess risk factors for an adverse outcome. The disease profile was classified using WHO pneumonia criteria, with tachypnoea or chest indrawing as defining clinical signs. Adverse outcome was defined as death, intensive care unit admission, tertiary care transfer or hospital stay $>10$ days.

Of 4206 admissions, 1758 (41.8\%) were classified as "no pneumonia" using WHO criteria and only 252 (6.0\%) met revised criteria for "severe pneumonia". The inpatient death rate was low $(0.4 \%$ of admissions $)$ with most deaths (11 out of $16 ; 68.8 \%$ ) occurring in the "severe pneumonia" group. An adverse outcome was recorded in $18.7 \%$ of all admissions and $60.7 \%$ of the "severe pneumonia" group. Children were hospitalised for a median of 7 days at an average cost of 253 USD per admission. Risk factors for adverse outcome included WHO-classified "severe pneumonia", age <1 year, low birth weight, previous recent admission with an acute respiratory infection and recent tuberculosis exposure. Breastfeeding, day-care attendance and pre-admission antibiotic use were associated with reduced risk.

Few hospital admissions met WHO criteria for "severe pneumonia", suggesting potential unnecessary hospitalisation and use of intravenous antibiotics. Better characterisation of the underlying diagnosis requires careful consideration. 


\section{Introduction}

Globally, pneumonia is the main cause of disease and death among young children outside the neonatal period [1]. In 2016, child pneumonia accounted for an estimated 650000 deaths, with the vast majority occurring in developing countries, including 57000 in Southeast and East Asia [1]. The World Health Organization (WHO) developed a clinical case management approach for use in resource-limited settings, aiming to reduce pneumonia-related deaths and improve access to life-saving antibiotics for children with bacterial pneumonia, while limiting unnecessary antibiotic use and reducing hospitalisations in those who can be safely managed as outpatients [2].

Although the WHO case management approach provides a simple strategy to reduce pneumonia-related mortality, it has important limitations. It may encourage unnecessary antibiotic use in children with viral infections [3] and lead to the misdiagnosis of asthma if a careful history of recurrent wheeze or night-time coughing is not solicited [4]. Despite these limitations, the WHO case management approach has had a positive impact [5], with studies crediting it with a 70\% reduction in under-5-year pneumonia-related mortality in some settings [6]. Although benefits are well documented in sub-Saharan Africa and South Asia [6], its clinical utility in East Asian settings, where many children present with "wheezy pneumonia" suggesting reactive airways disease [7,8], is not well described. Accurate diagnosis and disease severity assessment is important to limit unnecessary use of intravenous antibiotics and hospital admission. Careful characterisation of patients at risk of an adverse outcome is also important to prioritise these patients for more intensive management.

In Vietnam, pneumonia is the leading cause of paediatric hospital admission and places a huge burden on the healthcare system $[9,10]$. A retrospective study suggested that many "pneumonia" admissions may not be clinically justified [10], but case management practices in general are poorly documented. Detailed description of children admitted to hospital with "pneumonia" would provide a better understanding of the clinical utility of the WHO case management approach in Vietnam. Assessing pneumonia case management practices and risk factors for adverse outcomes would be informative to guide improved clinical care and public policy regarding resource allocation and vaccination schedules. The primary aim of this study was to describe the clinical characteristics and outcomes of children hospitalised with "pneumonia", as assessed by the admitting clinician, compared to revised WHO case definition criteria. A secondary aim was to assess social factors associated with an adverse outcome.

\section{Methods}

We conducted a prospective descriptive study of children hospitalised with "pneumonia" over a 1-year study period (July 1, 2017 to June 30, 2018). Written approval for the study was obtained from the Ethics Committee of Da Nang Hospital for Women and Children (January 13, 2017).

\section{Study setting}

The Da Nang Hospital for Women and Children is a secondary referral hospital in central Vietnam. The hospital has 900 beds with 570 beds reserved for children < 15 years old. Paediatric bed occupancy in 2017 was $>150 \%$. Annual reports suggest that $\sim 4000$ children with acute respiratory infections (ARIs) are admitted to the respiratory department and intensive care unit (ICU) every year. Children either present directly from home (through outpatient or emergency services) or are referred from district hospitals in Da Nang city or surrounding provincial hospitals in Quang Nam and Quang Ngai. Children with severe disease who do not improve on admission, including treatment in the local ICU, are referred to tertiary training hospitals in Ha Noi (The National Hospital of Pediatrics) or Ho Chi Minh City (Children's Hospitals number I and II). In 2016, the year preceding the study, ARIs accounted for nearly a third of paediatric hospital admissions to the Da Nang Hospital for Women and Children; 97\% were aged $<5$ years.

\section{Study population and procedures}

We recruited children aged 2-59 months admitted with a primary or secondary diagnosis of "pneumonia" as assessed by the admitting clinician. These children were routinely admitted to the respiratory department, or directly to the ICU. Independent researchers, not involved in clinical patient care, performed daily ward rounds in the respiratory department and the ICU to recruit new patients and record relevant clinical information.

Clinical information included respiratory signs and symptoms documented on admission, date of symptom onset, number of days from onset to admission, antibiotic use before admission, previous recent (during the past 2 weeks) admission with an ARI, recent (during the past 12 months) tuberculosis contact, gestational age (preterm if $<37$ weeks), birth weight (low if $<2500 \mathrm{~g}$ ) and pneumococcal vaccination (yes if received at least one dose). Social risk factors such as any breastfeeding, indoor biomass fuel (cooking with 
wood or charcoal) or cigarette smoke exposure (anyone smoking inside the house), day-care attendance or having an ill sibling at home were also documented. Details of all variables collected are summarised in supplementary table S1. Other clinical, laboratory and radiological information, as well as pneumonia outcome data, were updated intermittently during the period of hospital stay and finalised on discharge. A number of patients in a stable clinical condition were admitted to a private fee-paying ward; these patients were excluded from the study. Chest radiography was routinely performed in children admitted to hospital and assessed for consolidation by the lead investigator, using WHO working group definitions [11]. Hyperinflation was not documented.

\section{Pneumonia classification}

Children were classified based on revised WHO criteria using tachypnoea, defined as $\geqslant 50$ breaths $\cdot \mathrm{min}^{-1}$ if aged 2-11 months, or $\geqslant 40$ breaths $\min ^{-1}$ if aged $12-59$ months [12], and/or chest indrawing as defining clinical symptoms. Severe pneumonia was identified in the presence of any danger sign, including peripheral arterial oxygen saturation measured by pulse oximetry $\left(\mathrm{SpO}_{2}\right)<90 \%$ in room air, severe respiratory distress (grunting, nasal flaring), inability to drink or breastfeed, vomiting everything, lethargy or convulsions [2]. Table 1 provides an overview of the pneumonia classifications used and table 2 of the adverse outcome definition used.

\section{Statistical analysis}

Categorical data were tabulated and are presented as numbers and percentages. Their associations with WHO pneumonia criteria were determined using Chi-squared or Fisher's exact tests, as appropriate. "Wheeze heard with a stethoscope" was not recorded in the first 1038 cases, whereafter this was reliably recorded. For comparative analyses we considered unrecorded data as missing values and did not use any imputation. In Vietnam, children with respiratory symptoms often receive antibiotics before hospital presentation; we dichotomised the variables "time since symptom onset" and "duration of pre-admission antibiotics" using a clinically relevant threshold of 3 days.

Continuous data are summarised as mean \pm SD or median (interquartile range). Their associations with WHO-classified pneumonia were examined using ANOVA or Kruskal-Wallis tests for normally and non-normally distributed variables, respectively. We used uni- and multivariable logistic regression analyses to assess the value of pre-specified demographic and social factors associated with adverse outcome (as defined), including age, sex, low birth weight, WHO pneumonia classification, previous recent admission with an ARI, pre-admission antibiotic use, recent tuberculosis contact, breastfeeding, pneumococcal vaccination, cigarette or biomass smoke exposure and day-care attendance. Factors included in the multivariable model were selected based on a review of the published literature [13], as well as perceived clinical relevance and data quality. Post hoc analysis was done for breastfeeding stratified by age. Data analysis was carried out using SPSS (version 24.0; SPSS, Inc., Chicago, IL, USA). A p-value $<0.05$ was considered statistically significant. The strength of association was determined by estimating the odds ratio and $95 \%$ confidence interval.

\section{Results}

Figure 1 provides a flow diagram of patient enrolment. Of the 4206 children hospitalised with "pneumonia" as assessed by the admitting clinician, $41.8 \%$ failed to meet WHO pneumonia criteria by not having tachypnoea or chest indrawing on presentation. Table 3 provides an overview of patient demographics and pre-admission care in all children, classified according to revised WHO pneumonia

\begin{tabular}{|c|c|}
\hline $\begin{array}{l}\text { WHO pneumonia } \\
\text { classification }\end{array}$ & Symptoms and signs \\
\hline $\begin{array}{l}\text { No pneumonia } \\
\text { Pneumonia } \\
\text { Severe pneumonia }\end{array}$ & $\begin{array}{l}\text { No tachypnoea\# OR chest indrawing } \\
\text { Tachypnoea }{ }^{\#} \text { OR chest indrawing } \\
\text { Tachypnoea }{ }^{\#} \text { OR chest indrawing } \\
\text { PLUS any danger sign } \\
\text { - including } \mathrm{SpO}_{2}<90 \% \text { in room air, severe respiratory distress (grunting or } \\
\text { nasal flaring), inability to drink or breastfeed, vomiting everything, lethargy } \\
\text { or convulsions }\end{array}$ \\
\hline
\end{tabular}

WHO: World Health Organization; $\mathrm{SpO}_{2}$ : peripheral arterial oxygen saturation measured by pulse oximetry;

\#: rate of $\geqslant 50$ breaths $\cdot \mathrm{min}^{-1}$ aged $2-11$ months or $\geqslant 40$ breaths $\cdot \mathrm{min}^{-1}$ aged $12-59$ months [12]. 
TABLE 2 Overview of adverse outcome definition used

\begin{tabular}{ll} 
Adverse outcome definition $^{\#}$ & Description \\
\hline Death & Died in hospital \\
ICU admission & Admitted to ICU at any time during this admission \\
Tertiary care transfer & Transferred to tertiary care hospital \\
Prolonged admission & Hospital stay of $>10$ days
\end{tabular}

ICU: intensive care unit. ${ }^{\#}$ : composite outcome including death and/or ICU admission and/or tertiary transfer and/or prolonged admission.

criteria. Children aged $<2$ years accounted for $71.5 \%$ of the "pneumonia" admissions, with infants (aged $<1$ year) accounting for most (139 out of 252; 55.2\%) of the "WHO severe pneumonia" cases. Boys were more commonly admitted than girls $(58.8 \%$ boys; $\mathrm{p}<0.01)$ in all age categories, with the largest sex discrepancy observed among infants $(62.5 \%$ boys; $\mathrm{p}<0.01)$.

Table 4 summarises the clinical signs and symptoms observed. Ten cases with low oxygen saturation on admission did not meet WHO pneumonia criteria; these included children with sepsis $(n=4)$, congenital heart disease $(n=2)$, restrictive lung disease $(n=2)$, near drowning $(n=1)$ and primary pulmonary hypertension $(n=1)$. The four children transferred to a tertiary hospital with "WHO no pneumonia" included two children with restrictive lung disease, one with haemangioma causing airway obstruction and one with near drowning. Interestingly, 38.2\% of "WHO severe pneumonia" cases wheezed on auscultation and this was more common in cases with "WHO pneumonia" and "WHO severe pneumonia" compared to "WHO no pneumonia" (446 out of 2448 (18.2\%) versus 104 out of 1758 (5.9\%); p <0.001). Children with wheeze and no fever accounted for 24.2\% (61 out of 252) of "WHO severe pneumonia" cases, $15.6 \%$ (342 out of 2196) of the "WHO pneumonia" cases and 8.3\% (146 out of 1758) of the "WHO no pneumonia" cases. Few children were tested for HIV infection; of those tested two out of 79 (2.5\%) were HIV infected. Chest radiography was performed in 3971 children (94.4\%), with the majority $(52.6 \%)$ reported as normal and consolidation noted in $36.0 \%$.

Table 5 shows the outcome documented in children admitted with "pneumonia" as assessed by the admitting clinician, according to revised WHO pneumonia classification criteria. An adverse outcome was documented in $18.7 \%$ children. Most (60.7\%) adverse outcomes and the vast majority of deaths (seven out of nine; 77.8\%) occurred in children with "WHO severe pneumonia". The single child in the "WHO no pneumonia" category who died had cyanotic heart disease. The four deaths in children with "WHO

FIGURE 1 Flow diagram of patient enrolment. ICU: intensive care unit.

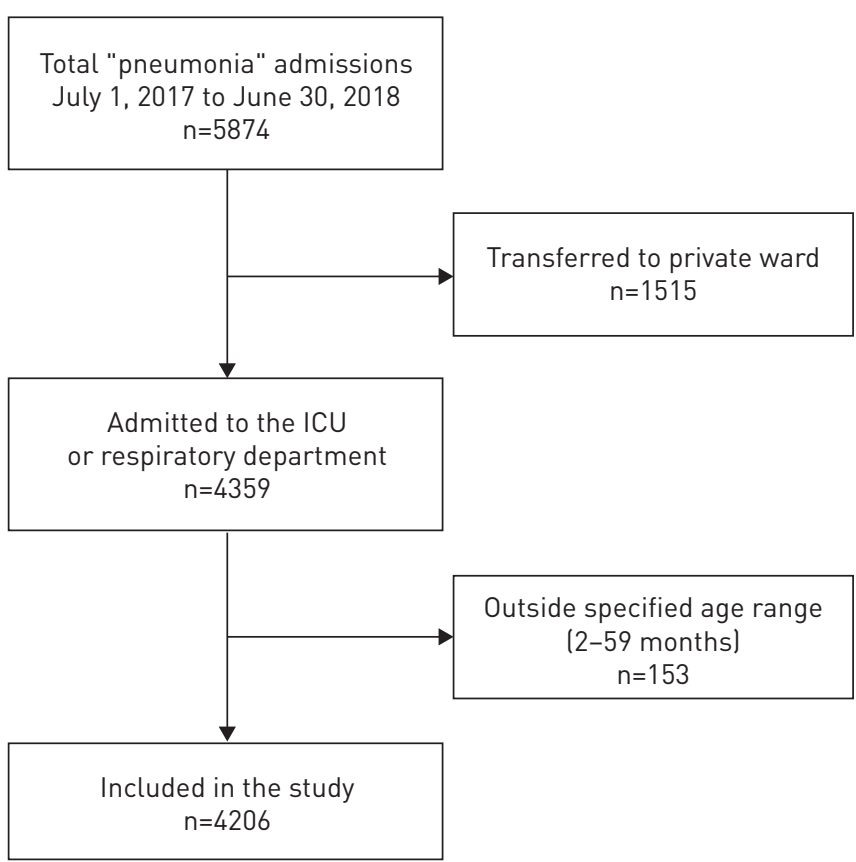




\begin{tabular}{|c|c|c|c|c|}
\hline \multirow[t]{2}{*}{ Demographics and pre-admission care } & \multicolumn{3}{|c|}{ WHO pneumonia classification } & \multirow[t]{2}{*}{ Total } \\
\hline & No pneumonia & Pneumonia & Severe pneumonia & \\
\hline $\begin{array}{l}\text { Subjects } \mathrm{n} \\
\text { Demographics }\end{array}$ & $1758(41.8)$ & $2196(52.2)$ & $252(6.0)$ & 4206 \\
\hline Age months & $18(10-28)$ & $16(10-24)$ & $10(5.5-17)$ & $16(9-25)$ \\
\hline 2-11 months & 589 (33.5) & $673(30.6)$ & $139(55.2)$ & $1401(33.3)$ \\
\hline $12-23$ months & $559(31.8)$ & $968(44.1)$ & 79 (31.3) & $1606(38.2)$ \\
\hline $24-59$ months & $610(34.7)$ & 555 (25.3) & 34 (13.5) & 1199 (28.5) \\
\hline Male sex & $1031(58.6)$ & $1301(59.2)$ & $142(56.3)$ & $2474(58.8)$ \\
\hline \multicolumn{5}{|l|}{ Pre-admission care } \\
\hline Private clinic & $533(30.3)$ & $657(29.9)$ & 55 (22.8) & $1245(29.6)$ \\
\hline Public clinic & $331(18.6)$ & $374(17.0)$ & 34 (13.5) & $739(17.6)$ \\
\hline Pharmacy & $202(11.5)$ & $301(13.7)$ & $22(8.7)$ & $525(12.5)$ \\
\hline Traditional healer & $14(0.8)$ & $19(0.9)$ & $1(0.4)$ & $34(0.8)$ \\
\hline Local hospital & $75(4.3)$ & 85 (3.9) & $26(10.3)$ & $186(4.4)$ \\
\hline None & 603 (34.3) & $760(34.6)$ & $114(45.2)$ & $1477(35.1)$ \\
\hline Referral from other hospital & $140(8.0)$ & $181(8.2)$ & $68(27.0)$ & $389(9.2)$ \\
\hline ARI readmission $\#$ & $182(10.4)$ & $261(11.9)$ & $63(25.0)$ & $506(12.0)$ \\
\hline Symptom duration days & $3(2-6)$ & $3(2-5)$ & $3(1-5)$ & $3(2-5)$ \\
\hline Symptom duration $<3$ days & 603 (34.3) & $807(36.7)$ & $114(45.2)$ & $1524(36.2)$ \\
\hline Pre-admission antibiotics & $894(50.9)$ & $1097(50.0)$ & $101(40.1)$ & $2092(49.7)$ \\
\hline Days of use & $3(2-5)$ & $3(2-5)$ & $3(2-5)$ & $3(2-5)$ \\
\hline Antibiotics duration $>3$ days & $386(43.2)$ & 387 (35.3) & 39 (38.2) & 812 (38.3) \\
\hline
\end{tabular}

Data are presented as $\mathrm{n}(\%)$ or median (IQR), unless otherwise stated. WHO: World Health Organization; IQR: interquartile range; ARI: acute respiratory infection. "\#: previous recent admission with an ARI and readmission within 2 weeks of discharge.

non-severe pneumonia" were attributed to sepsis (three) and acute pericarditis (one). Among children with "WHO no pneumonia" who were admitted for $>14$ days, 58.7\% (54 out of 92) were either referred from a district hospital with a clinical diagnosis of pneumonia and slow treatment response, or experienced a worsening of symptoms after hospital admission. Other children in this group had persistent wheezing or stridor $(n=10)$, chronic diarrhoea $(n=10)$ or an unrelated surgical problem $(n=28)$. Those who required assisted ventilation in the "WHO no pneumonia" group included children with clinical deterioration after admission $(n=8)$, sepsis $(n=1)$, hemangioma with airway obstruction $(n=1)$, congenital heart disease $(n=2)$, near drowning $(n=2)$ and acute pericarditis $(n=1)$.

Table 6 reflects uni- and multivariable analyses of factors associated with an adverse outcome. Children admitted with "WHO severe pneumonia" experienced the greatest risk (adjusted OR 7.4, 95\% CI 5.5-10.0). "WHO severe pneumonia", young age ( $<1$ year), male sex, low birth weight, recent tuberculosis contact, previous recent admission with an ARI and using biomass fuel for cooking were significantly associated with an adverse outcome. Protective factors included breastfeeding, especially in infants aged 2-11 months (OR 0.8 , 95\% CI $0.7-0.9$ on univariable analysis), older age, day-care attendance and pre-admission antibiotic use.

\section{DISCUSSION}

To our knowledge, this is the first study to provide a detailed characterisation of children admitted to hospital with "pneumonia" in Vietnam. In our study, nearly half of the children admitted to hospital did not meet WHO pneumonia classification criteria and few met the criteria for "WHO severe pneumonia"; these criteria generally guide hospital admission using the WHO case management approach. Previous pneumonia studies from Vietnam did not interrogate compliance with WHO classification criteria $[14,15]$, while potential over-diagnosis has been noted in a retrospective study from Vietnam [10] with similar concerns in other settings $[4,8]$.

The unnecessary use of intravenous antibiotics may be a particular problem in settings where this is regarded as routine practice and where hospitalisation is incentivised. However, over-diagnosis has also been observed with decentralised models of care if inadequate training is provided to frontline healthcare workers [16]. Our study findings are different to observations in sub-Saharan Africa where inadequate 


\begin{tabular}{|c|c|c|c|c|}
\hline \multirow[t]{2}{*}{ Clinical features } & \multicolumn{3}{|c|}{ WHO pneumonia classification } & \multirow[t]{2}{*}{ Total } \\
\hline & No pneumonia & Pneumonia & Severe pneumonia & \\
\hline \multicolumn{5}{|l|}{ Symptoms $\#$} \\
\hline Cough & $1607(91.4)$ & 2040 (92.9) & $204(81.0)$ & $3851(91.6)$ \\
\hline Fever & $1334(75.9)$ & $1725(78.6)$ & $163(64.7)$ & $3222(76.6)$ \\
\hline Runny nose & $421(23.9)$ & $508(23.1)$ & $37(14.7)$ & $966(23.0)$ \\
\hline Poor feeding" & $31(1.8)$ & 40 (1.9) & $7(2.8)$ & $78(1.9)$ \\
\hline Convulsions & $24(1.3)$ & $0(0)$ & $17(6.8)$ & $41(1.0)$ \\
\hline \multicolumn{5}{|l|}{ Signs $^{+}$} \\
\hline Tachypnoea & $0(0)$ & 2137 (97.3) & $228(90.5)$ & $2365(56.2)$ \\
\hline Chest indrawing & $0(0)$ & $517(23.5)$ & $201(79.8)$ & $718(17.1)$ \\
\hline Fever $\left(\geqslant 38.5^{\circ} \mathrm{C}\right)$ & $648(36.9)$ & $988(45.0)$ & $96(38.1)$ & $1732(41.2)$ \\
\hline $\mathrm{SpO}_{2}<90 \% \S$ & $10 / 67(14.9)$ & $0 / 323(0)$ & $131 / 188(69.7)$ & $141 / 578(24.4)$ \\
\hline Grunting & $1(0.1)$ & $0(0)$ & $105(41.7)$ & $106(2.5)$ \\
\hline Nasal flaring & $1(0.1)$ & $0(0)$ & 83 (32.9) & $84(2.0)$ \\
\hline Audible wheezing & 104 (5.9) & $387(17.6)$ & $59(23.4)$ & $550(13.1)$ \\
\hline Wheeze on auscultation ${ }^{\S}$ & $209 / 1301(16.1)$ & $535 / 1660$ (32.2) & $79 / 207(38.2)$ & $823 / 3168(26.0)$ \\
\hline Any wheeze without fever & 146 (8.3) & $342(15.6)$ & $61(24.2)$ & $549(13.1)$ \\
\hline Stridor & $5(0.3)$ & $22(1.0)$ & $4(1.6)$ & $(0.7)$ \\
\hline Lethargy & $7(0.4)$ & $0(0)$ & $28(11.1)$ & $35(0.8)$ \\
\hline \multicolumn{5}{|l|}{ Investigations } \\
\hline Abnormal chest radiography ${ }^{f}$ & $693 / 1638(42.3)$ & $1032 / 2087(49.4)$ & $157 / 246$ (63.8) & $1882 / 3971(47.4)$ \\
\hline Consolidation $\# \#$ & $254 / 693(36.7)$ & $352 / 1032(34.1)$ & $72 / 157(45.9)$ & $678 / 1882(36.0)$ \\
\hline \multicolumn{5}{|l|}{ Full blood count } \\
\hline$\geqslant 10$ neutrophils $\times 10^{9} \cdot \mathrm{L}^{-1}$ & $330 / 1721(19.2)$ & 422/2132 (19.8) & $67 / 249$ (26.9) & $819 / 4102(20.0)$ \\
\hline Haemoglobin $<100 \mathrm{~g} \cdot \mathrm{L}^{-1}$ & $285 / 1721(16.6)$ & $443 / 2133(20.8)$ & $72 / 249(28.9)$ & $800 / 4103(19.5)$ \\
\hline \multicolumn{5}{|l|}{ CRP $\mathrm{mg} \cdot \mathrm{L}^{-1}$} \\
\hline Median (IQR) & $18(6-48)$ & $21(6-36)$ & $16(6-32)$ & $18(6-36)$ \\
\hline$<10$ & $211 / 465(45.4)$ & $290 / 642(45.2)$ & $71 / 146(48.6)$ & $572 / 1253(45.7)$ \\
\hline $10-49$ & $151 / 465(32.5)$ & $220 / 642$ (34.3) & $49 / 146$ (33.6) & $420 / 1253$ (33.5) \\
\hline$\geqslant 50$ & $103 / 465$ (22.2) & $132 / 642(20.6)$ & $26 / 146$ (17.8) & $261 / 1253$ (20.8) \\
\hline
\end{tabular}

Data are presented as $\mathrm{n}(\%)$, unless otherwise stated. WHO: World Health Organization; $\mathrm{pO}_{2}$ : peripheral arterial oxygen saturation measured by pulse oximetry; CRP: C-reactive protein; IQR: interquartile range. ${ }^{\#}$ : reported by care giver; ${ }^{\eta}$ : not reliably captured; ${ }^{+}$: assessed on admission; ${ }^{\S}$ : in children in whom this feature was reliably recorded; ${ }^{f}$ : as assessed by the lead investigator using WHO endpoint criteria for consolidation [11]; ${ }^{\# \#}$ : using those with abnormal chest radiography results as the denominator.

healthcare access remains a major challenge and pneumonia-related mortality is high [17]. A multi-hospital retrospective cohort study from Kenya found that only 4\% (669 out of 16162) of "pneumonia" admissions did not meet WHO classification criteria [18]. This contrasts with findings in East Asia where healthcare access is generally good and pneumonia-related mortality low, with historical practice relying heavily on in-hospital treatment and the use of intravenous antibiotics [10].

Without careful interpretation of signs and symptoms, the use of WHO criteria may lead to a misclassification of common respiratory diseases such as bronchiolitis and asthma. A technical update to the WHO pneumonia guidelines recommends careful reconsideration in children with fast breathing who have wheezing without fever [19], which typically signifies reactive airway disease without bacterial infection. We found wheeze without fever in $>20 \%$ of children diagnosed with "WHO severe pneumonia". These children do not require antibiotics and misdiagnosis of asthma may delay optimal management [4]. Unnecessary antibiotic use in children with viral infections is a potential problem $[8,16]$, but a clear distinction between viral and bacterial pneumonia is highly problematic and even advanced diagnostic tests have major caveats [20]. Caregivers in Vietnam frequently access antibiotics without formal medical assessment [21] and this is common in most Asian countries: $>40 \%$ of children admitted with ARIs in Mongolia [22] and $>50 \%$ in the Philippines [23]. This is different to sub-Saharan Africa, where access to pre-admission antibiotics is more restricted [17]. 
TABLE 5 Outcome of children admitted to hospital with admitting clinician-diagnosed "pneumonia" in central Vietnam, according to WHO pneumonia classification criteria

\begin{tabular}{lcccc} 
Adverse outcome & \multicolumn{2}{c}{ WHO pneumonia classification } & \multirow{2}{*}{ Total } \\
\cline { 2 - 3 } & No pneumonia & Pneumonia & Severe pneumonia & \\
\hline Subjects n & 1758 & 2196 & 252 & 4206 \\
Overall adverse outcome & $236(13.4)$ & $399(18.2)$ & $153(60.7)$ & $788(18.7)$ \\
Died & $1(0.1)$ & $4(0.2)$ & $11(4.4)$ & $16(0.4)$ \\
ICU admission & $57(3.2)$ & $145(6.6)$ & $136(54.6)$ & $338(8.0)$ \\
$\quad$ Median number of days & $5(3-9)$ & $4(2-7)$ & $5.5(3-12)$ & $4(3-10)$ \\
Tertiary care transfer & $4(0.2)$ & $11(0.5)$ & $10(4.0)$ & $25(0.6)$ \\
Hospital stay >10 days & $213(12.1)$ & $319(14.5)$ & $97(38.5)$ & $629(15.0)$ \\
Hospital stay >14 days & $92(5.2)$ & $124(5.6)$ & $63(25.0)$ & $279(6.6)$ \\
$\quad$ Median number of days & $6(5-8)$ & $7(5-9)$ & $9(8-15)$ & $7(5-9)$ \\
0xygen supplementation & $67(3.8)$ & $224(10.2)$ & $174(69.0)$ & $465(11.1)$ \\
$\quad$ Median number of days & $2(1-5)$ & $2(1-4)$ & $3(1-6)$ & $2(1-5)$ \\
Assisted ventilation & $15(0.9)$ & $26(1.2)$ & $42(16.7)$ & $83(2.0)$ \\
$\quad$ Median number of days & $6(3-14)$ & $6(3-11)$ & $5(3-18)$ & $6(3-14)$ \\
Mean hospital cost USD & 112.5 & 121.5 & 515.7 & 253.1
\end{tabular}

Data are presented as $\mathrm{n}(\%)$ or median (interquartile range), unless otherwise stated. WHO: World Health Organization; ICU: intensive care unit. "\#: as defined in table 1.

Recent findings from the Pneumonia Etiology Research for Child Health (PERCH) project, performed in seven low- and middle-income countries, found that respiratory syncytial virus accounted for $>30 \%$ of chest radiography-confirmed pneumonia cases in children aged $<5$ years, while $<10 \%$ of cases were caused by Streptococcus pneumoniae [24]. Findings were consistent among the seven participating countries (Mali, the Gambia, Zambia, South Africa, Kenya, Bangladesh, Thailand) [25, 26]. A community-based study in Vietnam demonstrated that a point-of-care C-reactive protein (CRP) test can safely reduce unnecessary antibiotic use in children with an ARI [27], but we were unable to demonstrate significant differences in CRP or absolute neutrophil count values between different WHO pneumonia categories. In our study, almost a third of children with "WHO no pneumonia" had consolidation on chest radiography. This demonstrates the low sensitivity and specificity of clinical symptoms compared with chest radiography findings [28]. Several studies have also shown that $\sim 10 \%$ of patients with fever and an increased neutrophil count have radiographic consolidation, even in the absence of respiratory distress or auscultatory findings $[29,30]$.

Low birth weight, preterm birth, cigarette smoke exposure, indoor air pollution and poor vaccination uptake are well-recognised risk factors for child pneumonia [13, 31], but their correlation with adverse pneumonia outcome in central Vietnam has not been assessed. The observation that breastfeeding is protective in infancy is well documented [32]. We noted a trend suggesting protection from pneumococcal conjugate vaccine $(\mathrm{PCV})$, but this did not reach statistical significance because very few children were vaccinated. Despite good evidence from other settings [32, 33], PCV is not provided as part of the extended programme of immunisation in Vietnam. The fact that day-care attendance was inversely correlated with an adverse outcome suggests that self-limited viral infections may be more common in this group, or that these children have better access to early appropriate care. We found a significant association between biomass fuel use for home cooking and an adverse pneumonia outcome, which supports the existing literature [30], although the same was not observed for cigarette smoke exposure. The reliability of the information provided could not be verified, but the fact that cigarette smoke exposure was reported in $>50 \%$ of households is consistent with the high smoking prevalence among Vietnamese men [13].

The death rate among children admitted to hospital with pneumonia was low compared to other settings $[16,34,35]$, but similar to mortality rates recorded in Vietnam [10, 15]. Infants were over-represented among children with an adverse outcome, which is consistent with findings in other settings, even those with high PCV-13 vaccination coverage [36]. As in other studies, adverse outcomes were more frequently observed in boys than in girls $[9,15]$, which may be explained by delayed immunological maturity in boys [37]. Previous studies in Vietnam reported a "standard" hospital stay of 1 week following a pneumonia diagnosis $[10,38,39]$, which is consistent with our findings. However, a 3-5-day course of oral antibiotics has been shown to be adequate for non-severe pneumonia and hospitalisation for intravenous antibiotics may not be necessary [40]. In our study, the average cost of a pneumonia admission was 253.1 USD, 
TABLE 6 Uni- and multivariable analyses of demographic and social factors associated with adverse outcome (as defined in table 1) in children admitted to hospital with admitting clinician-diagnosed "pneumonia"

\begin{tabular}{|c|c|c|c|c|}
\hline \multirow[t]{2}{*}{ Factors } & \multicolumn{2}{|c|}{ Adverse outcome } & \multirow{2}{*}{$\begin{array}{l}\text { Univariable OR } \\
\text { (95\% CI) }\end{array}$} & \multirow{2}{*}{$\begin{array}{l}\text { Multivariable aOR } \\
(95 \% \mathrm{CI})\end{array}$} \\
\hline & $\mathrm{n} / \mathrm{N}$ & $\%$ & & \\
\hline \multicolumn{5}{|c|}{ WHO pneumonia classification } \\
\hline No pneumonia & $236 / 1758$ & 13.4 & 1 & 1 \\
\hline Pneumonia & $399 / 2196$ & 18.2 & $1.4(1.2-1.7)$ & $1.4(1.2-1.7)$ \\
\hline Severe pneumonia & $153 / 252$ & 60.7 & $10.0(7.5-13.3)$ & $7.4(5.5-10.0)$ \\
\hline \multicolumn{5}{|l|}{ Age } \\
\hline $2-11$ months & $388 / 1401$ & 27.7 & 1 & 1 \\
\hline $12-23$ months & $263 / 1606$ & 16.4 & $0.6(0.4-0.6)$ & $0.7(0.6-0.8)$ \\
\hline 24-59 months & $137 / 1199$ & 11.4 & $0.3(0.3-0.4)$ & $0.6(0.5-0.7)$ \\
\hline \multicolumn{5}{|l|}{ Sex } \\
\hline Female & $287 / 1732$ & 16.6 & 1 & 1 \\
\hline Male & $501 / 2474$ & 20.3 & $1.3(1.1-1.5)$ & $1.3(1.1-1.5)$ \\
\hline \multicolumn{5}{|l|}{ Breastfeeding $\#$} \\
\hline No & $84 / 319$ & 26.3 & 1 & 1 \\
\hline Yes & $704 / 3887$ & 18.1 & $0.6(0.5-0.8)$ & $0.8(0.6-1.0)$ \\
\hline \multicolumn{5}{|c|}{ Pneumococcal vaccination" } \\
\hline No & $700 / 4037$ & 17.3 & 1 & 1 \\
\hline Yes & $23 / 169$ & 13.6 & $0.7(0.4-1.1)$ & $0.9(0.6-1.5)$ \\
\hline \multicolumn{5}{|c|}{ Cigarette smoke exposure $^{+}$} \\
\hline No & $370 / 1994$ & 18.6 & 1 & 1 \\
\hline Yes & $418 / 2212$ & 18.9 & $1.0(0.9-1.2)$ & $1.0(0.8-1.1)$ \\
\hline \multicolumn{5}{|c|}{ Cooking with biomass fuel ${ }^{\S}$} \\
\hline No & $633 / 3418$ & 18.5 & 1 & 1 \\
\hline Yes & $191 / 788$ & 24.2 & $1.4(1.2-1.7)$ & $1.2(1.0-1.5)$ \\
\hline \multicolumn{5}{|c|}{ Low birth weight $(<2500 \mathrm{~g}$ ) } \\
\hline No & $679 / 3879$ & 17.5 & 1 & 1 \\
\hline Yes & $109 / 327$ & 33.3 & $2.4(1.9-3.0)$ & $1.8(1.3-2.5)$ \\
\hline \multicolumn{5}{|l|}{ Day-care attendance } \\
\hline No & $546 / 2097$ & 26.0 & 1 & 1 \\
\hline Yes & $242 / 2109$ & 11.5 & $0.4(0.3-0.4)$ & $0.6(0.5-0.7)$ \\
\hline \multicolumn{5}{|l|}{ ARI readmission ${ }^{f}$} \\
\hline No & $638 / 3700$ & 17.2 & 1 & 1 \\
\hline Yes & $150 / 506$ & 29.6 & $2.0(1.7-2.5)$ & $1.5(1.2-1.9)$ \\
\hline \multicolumn{5}{|c|}{ Antibiotic use before admission } \\
\hline No & $1763 / 3418$ & 51.6 & 1 & 1 \\
\hline Yes & $329 / 788$ & 41.8 & $0.7(0.6-0.8)$ & $0.8(0.7-0.9)$ \\
\hline \multicolumn{5}{|l|}{ Recent TB contact ${ }^{\# \#}$} \\
\hline No & $761 / 4117$ & 18.7 & 1 & 1 \\
\hline Yes & $27 / 89$ & 30.3 & $1.9(1.2-3.0)$ & $2.0(1.2-3.3)$ \\
\hline
\end{tabular}

ARI: acute respiratory infection; aOR: adjusted odds ratio; TB: tuberculosis; WHO: World Health Organization. " : any breastfeeding; ${ }^{\text {?: }}$ child received at least one dose; ${ }^{+}$: anyone smoking inside the house; §: cooking with wood or charcoal; ${ }^{f}$ : previous recent admission with an ARI and readmission within 2 weeks of discharge; ${ }^{\# \#}$ : TB contact within the last 12 months.

which is much higher than previous studies in Vietnam in which it ranged from 31 USD in 2006 to 158 USD in 2016 [10, 15, 38].

The main strength of our study is the fact that it represents the first detailed characterisation of children admitted with pneumonia in Vietnam, conducted as a prospective descriptive study of all admissions over a full calendar year. Our study was limited by the fact that we only enrolled patients in one secondary hospital in central Vietnam. However, we believe it provides a comprehensive overview of child pneumonia cases admitted to hospital in central Vietnam and complements a previous retrospective study that included primary, secondary and tertiary hospitals [10]. This retrospective study showed no significant difference in terms of the disease spectrum or outcome of ARI in the various hospitals [10]. The absence of information on children not admitted to hospital limits our ability to comment on the safety of the WHO case management approach. However, the admission threshold was generally very low 
and healthcare access in Da Nang is good, so it is considered unlikely that children excluded from this study would have deteriorated at home.

We were also limited by the extent of the microbiological work-up possible in the study setting and only report the results of standard blood tests performed on admission. Poor feeding was not recorded in a standardised fashion and it had to be excluded from our assessment of disease severity. In many hospitals the feeding of sick children receives inadequate attention. Some children who experienced an adverse outcome deteriorated after hospital admission, while the WHO classification is based on symptoms and signs at presentation, which explains some of the discrepancies. This illustrates potential pitfalls if any case management approach is applied without adequate clinical judgement. Children with respiratory symptoms may also require hospital admission for conditions other than pneumonia.

\section{Conclusion}

Although adoption of the WHO case management approach should reduce unnecessary hospital admissions and use of intravenous antibiotics, careful interpretation of signs and symptoms is required to ensure optimal management.

Acknowledgement: We acknowledge all patients and caregivers for their participation in the study. Especially, we give a big thank you to three nurses (Oanh T.K. Nguyen, Nhung T.M. Le, Ut T. Doan; Respiratory Dept, Da Nang Hospital for Women and Children), who helped with collecting patient data on admission.

Conflict of interest: None declared.

\section{References}

1 Troeger C, Blacker B, Khalil IA, et al. Estimates of the global, regional, and national morbidity, mortality, and aetiologies of lower respiratory infections in 195 countries, 1990-2016: a systematic analysis for the Global Burden of Disease Study 2016. Lancet Infect Dis 2018; 18: 1191-1210.

2 WHO. Revised WHO Classification and Treatment of Childhood Pneumonia at Health Facilities. Geneva, World Health Organization, 2014.

3 Hazir T, Qazi S, Nisar Y, et al. Assessment and management of children aged 1-59 months presenting with wheeze, fast breathing, and/or lower chest indrawing; results of a multicentre descriptive study in Pakistan. Arch Dis Child 2004; 89: 1049-1054.

4 Østergaard MS, Nantanda R, Tumwine JK, et al. Childhood asthma in low income countries: an invisible killer? Prim Care Respir J 2012; 21: 214-219.

5 Graham SM, English M, Hazir T, et al. Challenges to improving case management of childhood pneumonia at health facilities in resource-limited settings. Bull World Health Organ 2008; 86: 349-355.

6 Theodoratou E, Al-Jilaihawi S, Woodward F, et al. The effect of case management on childhood pneumonia mortality in developing countries. Int J Epidemiol 2010; 39: 155-171.

7 Addo-Yobo E, Anh DD, El-Sayed HF, et al. Outpatient treatment of children with severe pneumonia with oral amoxicillin in four countries: the MASS study. Trop Med Int Health 2011; 16: 995-1006.

8 Duke T. Pneumonia and bronchiolitis in developing countries. Arch Dis Child 2014; 99: 892-893.

9 Yoshida L-M, Suzuki M, Thiem VD, et al. Population based cohort study for pediatric infectious diseases research in Vietnam. Trop Med Health 2014; 42: S47-S58.

10 Nguyen TKP, Nguyen DV, Truong TNH, et al. Disease spectrum and management of children admitted with acute respiratory infection in Viet Nam. Trop Med Int Health 2017; 22: 688-695.

11 Cherian T, Mulholland EK, Carlin JB, et al. Standardized interpretation of paediatric chest radiographs for the diagnosis of pneumonia in epidemiological studies. Bull World Health Organ 2005; 83: 353-359.

12 WHO. Pocket Book of Hospital Care for Children: Guidelines for the Management of Common Childhood Illnesses. Geneva, World Health Organization, 2013.

13 Nguyen TKP, Tran TH, Roberts CL, et al. Risk factors for child pneumonia-focus on the Western Pacific Region. Paediatr Respir Rev 2017; 21: 95-101.

14 Toan N, Khe N, Höjer B. Expenditure and payment sources for treating acute respiratory infections in rural Vietnam. Southeast Asian J Trop Med Public Health 2001; 32: 682-688.

15 Tan KK, Dang DA, Kim KH, et al. Burden of hospitalized childhood community-acquired pneumonia: a retrospective cross-sectional study in Vietnam, Malaysia, Indonesia and the Republic of Korea. Hum Vaccin Immunother 2018; 14: 95-105.

16 Gowraiah V, Awasthi S, Kapoor R, et al. Can we distinguish pneumonia from wheezy diseases in tachypnoeic children under low-resource conditions? A prospective observational study in four Indian hospitals. Arch Dis Child 2014; 99: 899-906.

17 Enarson PM, Gie RP, Mwansambo CC, et al. Reducing deaths from severe pneumonia in children in Malawi by improving delivery of pneumonia case management. PloS One 2014; 9: e102955.

18 Agweyu A, Lilford RJ, English M, et al. Appropriateness of clinical severity classification of new WHO childhood pneumonia guidance: a multi-hospital, retrospective, cohort study. Lancet Glob Health 2018; 6: e74-e83.

19 WHO. Recommendations for Management of Common Childhood Conditions: Evidence for Technical Update of Pocket Book Recommendations: Newborn Conditions, Dysentery, Pneumonia, Oxygen Use and Delivery, Common Causes of Fever, Severe Acute Malnutrition and Supportive Care. Geneva, World Health Organization, 2012.

20 Zar HJ, Andronikou S, Nicol MP. Advances in the diagnosis of pneumonia in children. BMJ 2017; 358: j2739.

21 Hoa NQ, Chuc NTK, Phuc HD, et al. Unnecessary antibiotic use for mild acute respiratory infections during 28-day follow-up of 823 children under five in rural Vietnam. Tran R Soc Trop Med Hyg 2011; 105: 628-636. 
22 WHO. Antimicrobial Resistance in the Western Pacific Region: A Review of Surveillance and Health System Response. Geneva, World Health Organization, 2015.

23 Capeding MR, Bravo L, Santos J, et al. Prospective surveillance study of invasive pneumococcal disease among urban children in the Philippines. Pediatr Infect Dis 2013; 32: 383-389.

24 Morpeth SC, Deloria Knoll M, Scott JAG, et al. Detection of pneumococcal DNA in blood by polymerase chain reaction for diagnosing pneumococcal pneumonia in young children from low-and middle-income countries. Clin Infect Dis 2017; 64: Suppl. 3, S347-S356.

25 Hammitt LL, Kazungu S, Morpeth SC, et al. A preliminary study of pneumonia etiology among hospitalized children in Kenya. Clin Infect Dis 2012; 54: Suppl. 2, S190-S199.

26 Mermond S, Zurawski V, D’Ortenzio E, et al. Lower respiratory infections among hospitalized children in New Caledonia: a pilot study for the Pneumonia Etiology Research for Child Health project. Clin Infect Dis 2012; 54: Suppl. 2, S180-S189.

27 Do NT, Ta NT, Tran NT, et al. Point-of-care C-reactive protein testing to reduce inappropriate use of antibiotics for non-severe acute respiratory infections in Vietnamese primary health care: a randomised controlled trial. Lancet Glob Health 2016; 4: e633-e641.

28 Wingerter SL, Bachur RG, Monuteaux MC, et al. Application of the World Health Organization criteria to predict radiographic pneumonia in a US-based pediatric emergency department. Pediatr Infect Dis J 2012; 31: 561-564.

29 Shah S, Mathews B, Neuman MI, et al. Detection of occult pneumonia in a pediatric emergency department. Pediatr Emerg Care 2010; 26: 615-621.

30 Bachur R, Perry H, Harper MB. Occult pneumonias: empiric chest radiographs in febrile children with leukocytosis. Ann Emerg Med 1999; 33: 166-173.

31 Phuong NT, Hoang TT, Foster K, et al. Exploring pneumonia risk factors in Vietnamese infants: a survey of new mothers. BMJ Paediatr Open 2017; 1: e000155.

32 Sonego M, Pellegrin MC, Becker G, et al. Risk factors for mortality from acute lower respiratory infections (ALRI) in children under five years of age in low and middle-income countries: a systematic review and meta-analysis of observational studies. PloS One 2015; 10: e0116380.

33 Chisti MJ, Tebruegge M, La Vincente S, et al. Pneumonia in severely malnourished children in developing countries-mortality risk, aetiology and validity of WHO clinical signs: a systematic review. Trop Med Int Health 2009; 14: 1173-1189.

34 Hooli S, Colbourn T, Lufesi N, et al. Predicting hospitalised paediatric pneumonia mortality risk: an external validation of RISC and mRISC, and local tool development (RISC-Malawi) from Malawi. PloS One 2016; 11: e0168126.

35 Saha S, Hasan M, Kim L, et al. Epidemiology and risk factors for pneumonia severity and mortality in Bangladeshi children $<5$ years of age before 10 -valent pneumococcal conjugate vaccine introduction. BMC Public Health 2016; 16: 1233 .

36 le Roux DM, Myer L, Nicol MP, et al. Incidence and severity of childhood pneumonia in the first year of life in a South African birth cohort: the Drakenstein Child Health Study. Lancet Glob Health 2015; 3: e95-e103.

37 Muenchhoff M, Goulder PJ. Sex differences in pediatric infectious diseases. J Infect Dis 2014; 209: Suppl. 3, S120-S126.

38 Anh DD, Riewpaiboon A, Le Huu Tho S, et al. Treatment costs of pneumonia, meningitis, sepsis, and other diseases among hospitalized children in Viet Nam. J Health Popul Nutr 2010; 28: 436-442.

39 Nguyen PT, Tran HT, Truong HT, et al. Paediatric use of antibiotics in children with community acquired pneumonia: a survey from Da Nang, Vietnam. J Paediatr Child Health 2019, in press [https://doi.org/10.1111/jpc. 14413].

40 Ginsburg AS, May SJ, Nkwopara E, et al. Methods for conducting a double-blind randomized controlled clinical trial of three days versus five days of amoxicillin dispersible tablets for chest indrawing childhood pneumonia among children two to 59 months of age in Lilongwe, Malawi: a study protocol. BMC Infect Dis 2018; 18: 476. 